\title{
Glycation cross-links inhibit matrix metalloproteinase-2 activation in vascular smooth muscle cells cultured on collagen lattice
}

\author{
M. Kuzuya, T. Asai, S. Kanda, K. Maeda, X. W. Cheng, A. Iguchi \\ Department of Geriatrics, Nagoya University Graduate School of Medicine, 65 Tsuruma-cho, Showa-ku, Nagoya, Japan
}

\begin{abstract}
Aims/hypothesis. Extracellular matrix glycation has been proposed to contribute to the arterial stiffness observed in aging and diabetes. We examined whether matrix protein glycation regulates the proleolytic process through the manipulation of matrix metalloproteinases (MMPs) activation, using collagen fibrils model.

Methods. Vascular smooth muscle cells were cultured on control or glycated collagen fibrils. Matrix metalloproteinase- 2 activation and the production of tissue inhibitors of metalloproteinase (TIMPs) were measured in the conditioned medium by using gelatin zymography and immunoblotting. Membrane type 1 matrix metalloproteinase (MT1-MMP) expression was also measured in cell lysates.

Results. When smooth muscle cells were cultured on collagen fibrils, pro-MMP-2 processing to active form was observed in the conditioned medium in coincidence with the increased MT1-MMP expression and the suppressed TIMP-2 production. Culturing
\end{abstract}

smooth muscle cells on glycated collagen fibrils inhibited MMP-2 activation and attenuated MT1-MMP expression without the alteration of TIMP-2 production compared with control fibrils, indicating the possible mechanism of the suppression of MT1-MMP expression for the inhibition of MMP-2 activation on glycated collagen fibrils. Inclusion of aminoguanidine, an inhibitor of cross-linking formation, during collagen glycation restored the MMP-2 activation, suggesting the role of cross-links on the inhibition of MMP-2 activation.

Conclusion/interpretation. These observations suggest that glycation-induced cross-linking formation in interstitial collagen contributes to arterial stiffness in aging and diabetes through the manipulation of matrix metalloproteinase activation along with the reduction of the susceptibility to proteolytic enzymes. [Diabetologia (2001) 44: 433-436]

Keywords Matrix metalloproteinase-2, membrane type 1 matrix metalloproteinase, glycation, smooth muscle cell, collagen.
With aging or in diabetes, arteries exhibit increases in media thickness and wall collagen content with stiff-

Received: 16 October 2000 and in revised form: 18 December 2000

Corresponding author: M. Kuzuya, MD, Ph D; Department of Geriatrics, Nagoya University Graduate School of Medicine, 65 Tsuruma-cho, Showa-ku, Nagoya 466-8550, Japan

Abbreviations: AGEs, advanced glycation end products; MMPs, matrix metalloproteinases; TIMPs, tissue inhibitors of metalloproteinases; SMCs, smooth muscle cells; MT1-MMP, membrane type 1 matrix metalloproteinases; AGE-BSA, glycated bovine serum albumin; G6-P, glucose 6-phosphate. ening of the vessel wall $[1,2]$. The major proposed mechanisms for the development of arterial stiffness are the accumulation of additional arterial interstitial collagen and advanced glycation end products (AGEs)-dependent cross-links [3]. AGE-mediated cross-links lead to a decrease in the susceptibility of extracellular matrix to enzymatic degradation [3]. Thus, AGEs might be implicated in an increase in collagen content in arterial wall, artery stiffness, and tissue rigidity through conferring a high resistance to matrix degradation by proteolytic enzymes, such as matrix metalloproteinases (MMPs). Matrix metalloproteinases are zinc-dependent endopeptidases that 


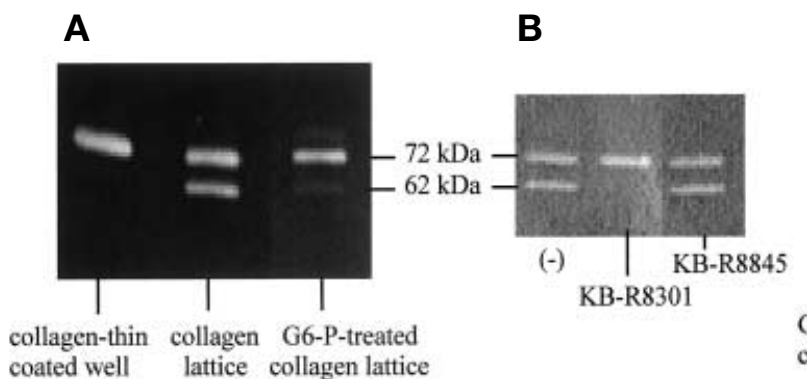

coated well lattice collagen lattice
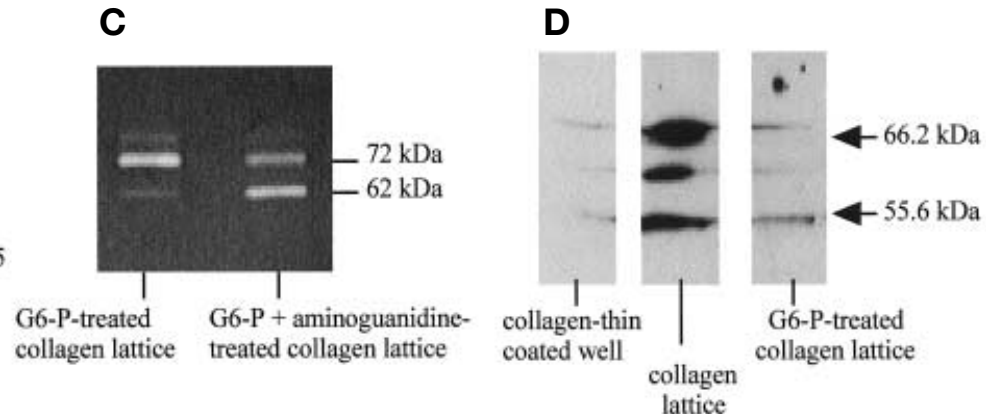

Fig.1(A-D). Zymography analysis of conditioned media from SMCs and effect of collagen lattice on MT1-MMP expression. Conditioned media from $48 \mathrm{~h}$ culture of SMCs on various substrates were analysed by gelatin zymography. SMCs were cultured on collagen thin-coated wells, collagen gel, G6-P-treated (glycated) collagen lattice (A), or on collagen lattice in the presence or absence of KB-R8301 (10 $\mu \mathrm{mol} / \mathrm{l})$, a MMP inhibitor, or KB-R8845 (10 $\mu \mathrm{mol} / \mathrm{l})$, a control compound $(\mathbf{B})$, or on collagen lattice, which was pretreated with G6-P in the presence or absence of aminoguanidine (C). (D); SMCs were cultured on collagen thin-coated well, collagen lattice, or glycated collagen lattice for $48 \mathrm{~h}$. Cell lysates were then electrophoresed in $10 \%$ SDS-polyacrylamide gel under reducing condition and immunoblotted for MT1-MMP. Arrows indicate standard marker proteins

are secreted in a latent form (pro-MMP) requiring proteolytic processing in order to become catalytically active [4]. The activation of pro-MMP-2, which is constitutively produced by vascular smooth muscle cells (SMCs) [5], is triggered by an interaction between pro-MMP-2 and cell surface bound membrane type 1 MMP (MT1-MMP) [6].

We hypothesized that AGE-mediated cross-links of matrix protein might also regulate the proleolytic process through manipulating the activation of MMPs, resulting in the inhibition of extracellular matrix protein degradation. We examined whether glycation might affect SMCs proteolytic balance on collagen lattice through the inhibition of pro-MMP-2 processing to active form.

\section{Materials and methods}

Collagen lattice and glycation. A neutralized collagen type I solution $(1.5 \mathrm{mg} / \mathrm{ml}$, Collaborative Research, Inc. Bedford, Mass., USA) was allowed to gel in the plates for $1 \mathrm{~h}$ at $37^{\circ} \mathrm{C}$. The gels were incubated with or without glucose 6phosphate (G6-P, $0.2 \mathrm{~mol} / \mathrm{l}$, Sigma Chemical Co., St. Louis, $\mathrm{Mo}, \mathrm{USA})$ in $0.2 \mathrm{~mol} / \mathrm{l}$ phosphate buffer $(\mathrm{pH} 7.8)$ in the presence or absence of aminoguanidine, an inhibitor of AGE and cross-linking formation, $(0.25 \mathrm{~mol} / \mathrm{l}$, Tokyokasei, Tokyo, Japan) for 2 weeks at $37{ }^{\circ} \mathrm{C}$ [7]. To prepare collagen thin coating, collagen solution in $0.02 \mathrm{~N}$ acetic acid $(100 \mu \mathrm{g} / \mathrm{ml})$ was added into plates and incubated overnight at $4{ }^{\circ} \mathrm{C}$. A G 6-P was used instead of glucose to speed up the process of nonenzymatic glycation.
Cell culture and preparation of conditioned media. Bovine SMCs derived from fetal bovine aorta were isolated [5] and cultured in DMEM containing $10 \%$ fetal calf serum and antibiotics. Experiments were performed with SMCs at passage 6-10. Cultured human coronary SMC was obtained from $\mathrm{Ku}-$ rabo Industries, LTD. (Tokyo, Japan). SMCs suspended in DMEM $\left(10^{6}\right.$ cells $\left./ \mathrm{ml}\right)$ were seeded onto collagen gels and cultured at $37^{\circ} \mathrm{C}$ for $48 \mathrm{~h}$. In some experiments, SMCs were cultured in the presence of KB-R8301 $(10 \mu \mathrm{mol} / \mathrm{l})$, a hydroxamic acid-based MMP inhibitor, or KB-R8845 (10 $\mu \mathrm{mol} / \mathrm{l})$, a nonhydroxamic acid control compound [5]. The conditioned media were used for the gelatin zymography. In some experiments, conditioned media were concentrated by 10 fold for the western blot analysis of TIMPs.

Attachment assay. For the attachment assay, we used human SMCs, since blocking antibodies against bovine integrins are not available. The human SMC suspension in Dubecco's modified Eagle's medium (DMEM) containing 0.3\% BSA was preincubated with or without blocking antibodies against integrin $(1: 200, \mathrm{vol} / \mathrm{vol})$ or glycated BSA (AGE-BSA, $500 \mu \mathrm{g} / \mathrm{ml})$ for $30 \mathrm{~min}$, and plated at $1.5 \cdot 10^{4}$ cells $/ 100 \mu$ l onto collagen gels in 96-well plates. We prepared AGE-BSA as described previously $[7,8]$. After $1 \mathrm{~h}$ incubation at $37^{\circ} \mathrm{C}$, the number of attached cells was counted as described previously [8].

Gelatin zymography and western blot analysis. Conditioned medium was analysed by gelatin zymography [5, 9]. Smooth muscle cell lysates and concentrated conditioned medium were used for the western blot analysis of MT1-MMP and TIMPs, respectively [9]. Quantitative results of the assays were obtained by densitometry.

Reagents. Rabbit anti-TIMP-1, 2 polyclonal antibodies, and rabbit anti-MT1-MMP polyclonal antibody were obtained from Sigma (Tokyo, Japan). Anti-human integrin $\beta 1$ monoclonal antibody (clone $\mathrm{P} 4 \mathrm{C} 10$ ) and anti-human integrin $\alpha 2$ monoclonal antibody (clone P1E6) were obtained from Gibco BRL (Tokyo, Japan). The KB-R8301 and KBR8845 were kindly provided by Dr. K. Yoshino (R \& D Lab. Nippon Organon K.K., Osaka, Japan).

Statistical analysis. Results are given as means \pm SE. Data were analysed by unpaired Student's $t$ test on raw data using StatView software (Abacus Concepts). A $p$ value of less than 0.05 was considered statistically significant.

\section{Results}

Gelatin zymographic analysis of the conditioned medium from $48 \mathrm{~h}$ culture of SMCs on collagen thin-coated well 

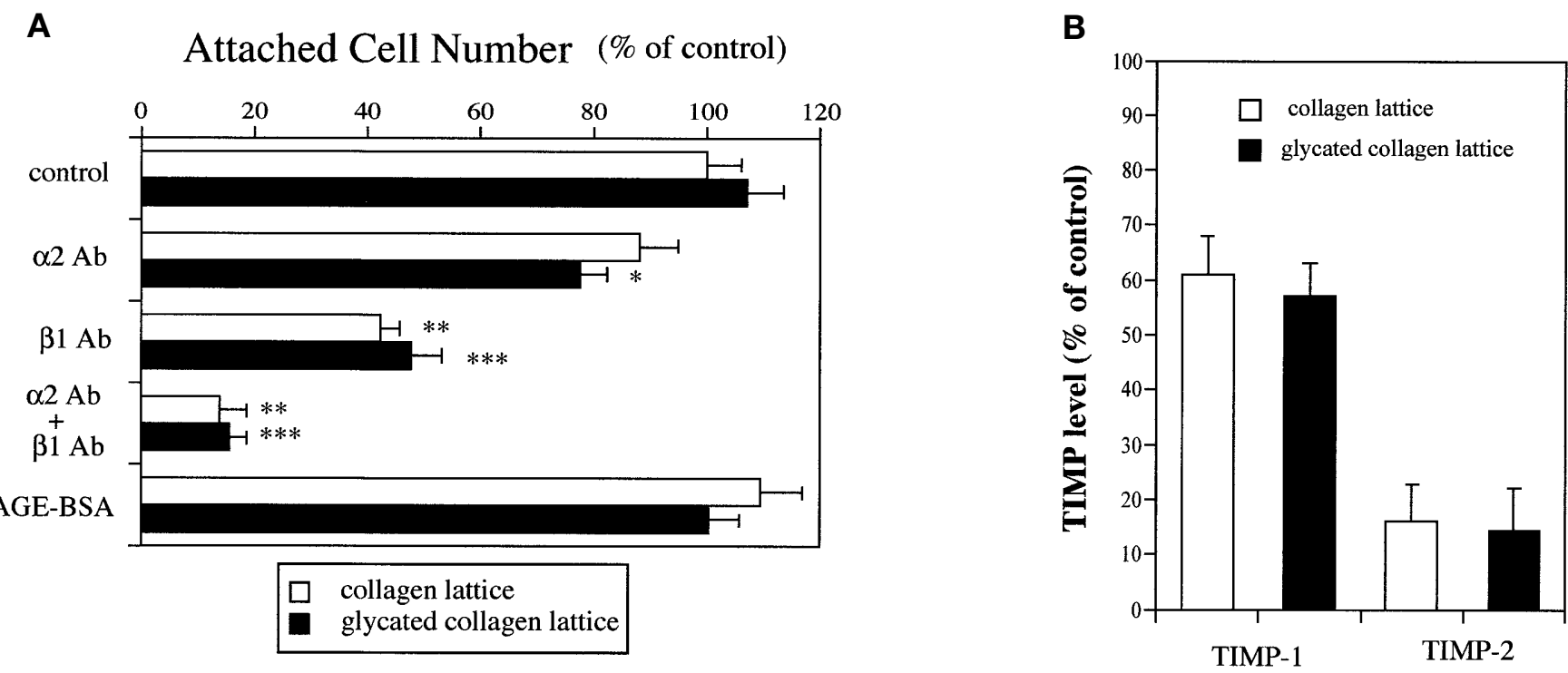

Fig. 2 A, B. Effect of anti-integrin antibodies on SMCs attachment on collagen lattice (A) and effect of collagen lattice on TIMPs production (B). (A); SMCs were plated on control or glycated collagen lattice in the presence or absence of blocking anti-integrin antibodies (1:200, vol/vol) or AGE-BSA $(500 \mu \mathrm{g} / \mathrm{ml})$. After $1 \mathrm{~h}$ incubation, unattached cells were washed and the number of attached cells was measured. Data are expressed as means $\pm \operatorname{SE}(n=8)$ and are a percentage of the number of cells attached on control collagen lattice. $* p<0.01$ vs control on glycated collagen lattice. $* * p<0.001$ vs control on collagen lattice. $p<0.001$ vs control on glycated collagen lattice. (B); SMCs were cultured on collagen thin-coated well, collagen lattice, or glycated collagen lattice for $48 \mathrm{~h}$. Tenfold-concentrated conditioned media were applied for immunoblotting of TIMP-1 and 2. Quantitative results of TIMPs were obtained by densitometry of the immunoblots. Results are expressed as a percentage of conditioned medium from collagen thin-coated well. The means $\pm \mathrm{SE}$ of four independent experiments are shown $(\mathbf{A}, \mathbf{B}) \square$, collagen lattice; glycated collagen lattice

showed that major gelatinolytic activity was observed at $72 \mathrm{k}$ $\mathrm{Mr}$, which was most likely to correspond to the inactive form of MMP-2 (Fig. 1A). Culturing on collagen lattice stimulated processing of pro-MMP-2 to a molecular weight $62 \mathrm{kMr}$, an active form of MMP-2. This pro-MMP-2 processing was also observed in the conditioned medium from human coronary SMCs cultured on collagen lattice (data not shown). Incubation of SMCs on collagen lattice with the MMP inhibitor, KB-R8301, effectively blocked pro-MMP-2 processing (Fig. 1B). Whereas the control compound, KB-R8845, had no effect (Fig.1B). Only $72 \mathrm{kMr}$, pro-MMP-2, was detected from culture on glycated collagen lattice (Fig. 1A). Inclusion of aminoguanidine during glycation reaction restored the band at $62 \mathrm{kMr}$, an active form of MMP-2, in the conditioned medium (Fig. 1C).

A blocking anti- $\beta 1$ integrin antibody and the combination of $\alpha 2$ and $\beta 1$ antibodies decreased SMC attachment on collagen lattice to $40 \%$ and $15 \%$, respectively (Fig. 2A). There was no difference in the effect of these antibodies on SMC attachment on glycated collagen lattice compared with those on control collagen lattice. Smooth muscle cell attachment on gly- cated and on control collagen lattice was not affected in the presence of excess AGE-BSA.

Immunoblots of the extracts of SMCs cultured on collagen thin-coated well showed faint immunoreactive bands of 66-68 $\mathrm{kMr}, 63 \mathrm{kMr}$, comparable to pro-MT1-MMP and activated MT1-MMP, respectively, as well as $55 \mathrm{kMr}$, corresponding to its degradation product (Fig.1D). Culturing SMCs on collagen lattice induced a dramatic increase in these immunoreactive bands. Whereas the expression of these bands was reduced in the cell lysates from cultures on glycated collagen gel.

Culturing SMCs on collagen lattice reduced TIMP-1 and TIMP-2 production, compared with that on collagen thin-coated well (Fig. 2B) No difference in production was observed between cultures on control and glycated collagen lattice (Fig. 2B).

\section{Discussion}

Matrix metalloproteinase-2 is secreted as an inactive proenzyme, which is activated by a membrane-linked process that involves MT1-MMP and TIMP-2. Complexed with TIMP-2, MT1-MMP has been found to serve as a cell surface receptor for MMP-2. The activation of bound MMP-2 has been proposed to occur through an initial cleavage by other MT1-MMP molecules to an active form [6,9]. Although TIMP-2 plays a role in the activation of MMP-2, an excess of TIMP-2 inhibits MMP-2 activation by binding all MT1-MMP molecules. Thus, the activity of MMP-2 is regulated, and not only is it dependent on de novo synthesis of the latent enzyme, but it also appears to be determined by activation of the zymogen and by the presence of the specific inhibitor, TIMP-2.

In the present study, we showed that culturing SMCs on collagen fibrils induced pro-MMP-2 processing to an active form of MMP-2, which was accompanied with SMCs MT1-MMP expression and processing. Culturing SMCs on collagen lattice caused a reduction of TIMP-2 production. The 
TIMP-2, is however, not likely to be involved in the suppression of pro-MMP-2 activation on glycated collagen lattice, since no detectable changes in TIMP-2 production were observed in the conditioned medium from SMCs cultured on glycated lattice. The expression of MT1-MMP was decreased in SMCs cultured on glycated collagen fibrils, suggesting that the suppression of MT1-MMP expression is mainly responsible for the decreased MMP-2 activation on glycated collagen fibril.

The results that the interaction of SMCs integrins with collagen molecule is not altered by the glycation and that AGE molecules has no effect on SMCs interaction with glycated collagen molecule suggest that the inhibition of MMP-2 activation on glycated collagen fibrils is not due to simple changes in cell-collagen molecule interaction through integrins. We showed that cross-linking formation is essential for the inhibition of MMP-2 processing on collagen fibrils, because inclusion of aminoguanidine during the glycation reaction completely recovered this effect of glycation. In addition, the treatment of collagen fibrils with glutaraldehyde, a well-known chemical cross-linker, also inhibited MMP-2 activation on collagen fibrils (data not shown). The biological effects of matrix components vary greatly depending on their structural configuration and physical characteristics. It has been proposed that mechanical interactions between cells and extracellular matrix modulate cell behaviors [10]. Thus, it is possible that altering the mechanical integrity of fibrillar collagen by glycation-induced cross-linking formation contributes to the inhibition of pro-MMP-2 processing on glycated collagen fibrils. This concept is supported by the fact that pro-MMP-2 processing was not detected in the conditioned medium from cultured SMCs on rigid collagen thin-coated plastic well.

In conclusion, we showed that AGE-induced cross-linking formation in collagen fibrils inhibits MMP-2 activation through the attenuation of SMC MT1-MMP expression. This decreased proleolytic activity induced by the SMC-cross-linked collagen fi- brils interaction might help explain, at least in part, the decrease in glycated matrix protein remodelling along with its resistance of susceptibility to proteolytic enzymes contributing to the development of arterial stiffness.

Acknowledgements. We thank T. Shibata for her expert technical assistance. We are grateful to Dr. M. A. Ramos for his critical review.

\section{References}

1. Gerity RG, Cliff WJ (1972) The aortic tunica intima in young and aging rats. Exp Mol Pathol 16: 382-402

2. Oxlund H, Rasmusen LM, Andreassen TT, Heickendorff L (1989) Increased aortic stiffness in patients with type I (insulin-dependent) diabetes mellitus. Diabetologia 32: 745-752

3. Brownlee M, Vlassara H, Kooney A, Ulrich P, Cerami A (1986) Aminoguanidine prevents diabetes-induced arterial wall protein cross-linking. Science 232: 1629-1632

4. Dollery CM, McEwan JR, Henney AM (1995) Matrix metalloproteinases and cardiovascular disease. Circ Res 77: 863-868

5. Kanda S, Kuzuya M, Ramos MA et al. (2000) Matrix metalloproteinase and $\alpha v \beta 3$ integrin-dependent vascular smooth muscle cell invasion through a type I collagen lattice. Arterioscler Thromb Vasc Biol 20: 998-1005

6. Sato H, Takino T, Okada Y, Shinagawa A, Yamamoto E, Seiki M (1994) A matrix metalloproteinase expressed on the surface of invasive tumor cells. Nature 370: 61-65

7. Kuzuya M, Satake S, Ai S et al. (1998) Inhibition of angiogenesis on glycated collagen lattices. Diabetologia 41: 491-499

8. Kuzuya M, Satake S, Miura H, Hayashi T, Iguchi A (1996) Inhibition of endothelial cell differentiation on a glycosylated reconstituted basement membrane complex. Exp Cell Res 226: 336-345

9. Koike T, Kuzuya M, Asai T et al. (2000) Activation of MMP-2 by Clostridium difficile toxin B in bovine smooth muscle cells. Biochem Biophys Res Commun 277: 43-46

10. Ingber DE, Folkman J (1989) Mechanochemical switching between growth and differentiation during fibroblast growth factor-stimulated angiogenesis in vitro: role of extracellular matrix. J Cell Biol 109: 317-330 